\title{
MicroReview
}

\section{Mycobacterium tuberculosis phagosome}

\author{
V. Deretic* and R. A. Fratti \\ Department of Microbiology and Immunology, University \\ of Michigan Medical School, Medical Sciences Building II, \\ Ann Arbor, MI 48109-0620, USA.
}

\section{Summary}

The arrest of Mycobacterium tuberculosis phagosome maturation in infected macrophages is a phenomenon of dual significance both for the pathogenesis of tuberculosis and as a model system to study interference of microbes with membrane trafficking and organelle biogenesis in host cells. Among other factors, compartment-specialized regulators of vesicular trafficking and other parts of membrane fusion machinery are likely to play a role in these processes. Here we summarize the emerging view of mycobacterial phagosome maturation arrest in the context of the dynamic processes of intracellular membrane trafficking.

\section{Introduction}

Mycobacterium tuberculosis is a potent human pathogen infecting close to one-third of the world's population (World Health Organization Tuberculosis Fact Sheet; http://www. who.int). Both the bacillus and the host participate in a complex set of interactions that can lead to elimination of the pathogen (no infection), long-term containment of the bacilli (latent infection) or active disease (primary or reactivation tuberculosis). Central to the ability of $M$. tuberculosis to infect the host and cause active or latent disease is the propensity of the tubercle bacillus, and some other mycobacterial pathogens, to enter the host mononuclear phagocytic cells and survive and multiply within macrophages (Fig. 1). In their seminal studies, Armstrong and Hart (1971) have established that $M$. tuberculosis phagosomal compartments do not mix with ferritin-labelled lysosomes, thus defining one of the central paradigms of $M$. tuberculosis pathogenesis, referred to as the inhibition of phagosomelysosome fusion. More recent studies have confirmed that members of the $M$. tuberculosis complex (e.g. virulent $M$. tuberculosis and the vaccine strain $M$. bovis BCG) reside

Received 9 October, 1998; revised 16 November, 1998; accepted 20 November, 1998. *For correspondence. E-mail Deretic@umich.edu; Tel. (+1) 734 7631580; Fax (+1) 7346476243. in a privileged phagosomal compartment, remaining sequestered away from the terminal endocytic organelles $(\mathrm{Xu}$ et al., 1994; Clemens and Horwitz, 1995; Hasan et al., 1997; Via et al., 1997). The exact mechanisms underlying this phenomenon are not completely understood, although significant progress has been made in defining the macrophage receptors participating in $M$. tuberculosis uptake, delineating the characteristics of the compartments that the bacillus occupies upon phagocytosis, and uncovering several aspects of its interference with fundamental membrane trafficking processes in eukaryotic cells.

\section{Entry and early events during M. tuberculosis phagocytosis}

A number of ligand-receptor interactions mediate the entry of mycobacteria into the macrophage (Ernst, 1998). Complement receptors are believed to play a significant role in the efficient uptake of the bacillus by host macrophages (Schlesinger et al., 1990). Mycobacterial opsonization depends on the alternative complement pathway (Schlesinger et al., 1990) and another, recently described, unconventional pathway of C3b formation involving a mycobacterial cell wall component (Schorey et al., 1997). The relative contributions of macrophage receptors [e.g. complement receptors CR1, CR3 and CR4, mannose receptor, surfactant protein receptors, CD14, scavenger receptors and Fc $\gamma$ receptors; recently reviewed by Ernst (1998)], opsonic and non-opsonic interactions of mycobacterial ligands with macrophage receptors and whether the route of entry of $M$. tuberculosis affects its intracellular fate by determining phagosomal characteristics remain unsettled issues (Ernst, 1998).

Uptake of M. tuberculosis by the macrophage is associated with a number of early intracellular signalling events, i.e. recruitment of and activation of Src family protein tyrosine kinases (PTKs) both within the cytoskeletal (Triton $\mathrm{X}-100$ insoluble) and cytosolic fractions, increased tyrosine phosphorylation of multiple macrophage proteins, and activation of phospholipase D (PLD) (Kusner et al., 1996). These events are consistent with receptor-mediated phagocytosis and are reminiscent of the processes associated with cross-linking of complement receptors. Activation of PTK appears to enhance stimulation of PLD activity and the associated increase in phosphatidic acid (PA). PA 


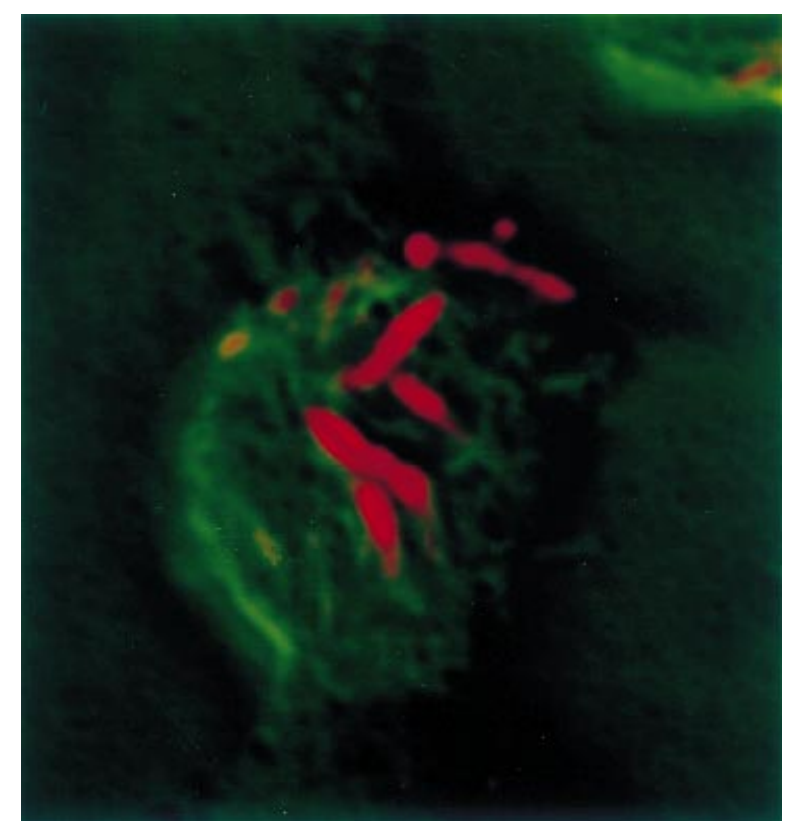

Fig. 1. Laser scanning confocal microscopy of $\mathbf{J} 774$ cells infected with GFP-expressing $M$. bovis BCG. Shown are confocal optical sections with merged Nomarski differential interference contrast (green pseudocolour) and GFP fluorescence (red pseudocolour) images.

may trigger a number of downstream processes necessary for membrane remodelling during phagocytosis and can cause activation of defence functions such as NADPH oxidase. Furthermore, PA, other acidic phospholipids and sitedirected modifications of lipids have become increasingly important in general vesicular trafficking and membrane budding at the stage of the recruitment of various factors controlling such processes (De Camilli et al., 1996). However, no differences have been detected when virulent $M$. tuberculosis was compared with opsonized zymosan (Kusner et al., 1996), and thus it is possible that other processes concomitant with or further downstream from these events determine the fate of mycobacterial phagosomes.

\section{Reduced acidification of mycobacterial phagosomes}

The phagosomes containing M. tuberculosis, M. bovis BCG and Mycobacterium avium display significantly reduced acidification (Crowle et al., 1991; Oh and Straubinger, 1996; Hackam et al., 1998) compared with killed organisms or model phagosomes containing inanimate objects (e.g. latex beads). This has been associated with an apparent paucity or lack of $\mathrm{H}^{+}$-ATPase pumps on these vacuoles (Strugill-Koszycki et al., 1994; Xu et al., 1994) (Table 1). A considerable heterogeneity of $\mathrm{pH}$ among different vesicles (Oh and Straubinger, 1996) and a detectable initial drop in $\mathrm{pH}$, followed by a recovery and equilibration at $\mathrm{pH}$ of 6.36.5 , have been noted (Strugill-Koszycki et al., 1994; Schaible et al., 1998). In a more recent study, alternative sources of protons (other than $\mathrm{H}^{+}$-ATPase) have been considered, and the $\mathrm{Na}^{+} / \mathrm{H}^{+}$exchanger was found to be incorporated into phagosomal membranes in macrophages (Hackam et al., 1997). However, the effect of this antiporter on acidification is dependent upon the concentration of lumenal $\mathrm{Na}^{+}$available for exchange with protons, and direct measurements suggest that its contributions to $\mathrm{pH}$ of model phagosomes and $M$. bovis BCG phagosomal compartments may be negligible (Hackam et al., 1998). Somewhat surprisingly, specific inhibitors of the vacuolar proton-ATPase abrogate the mild acidification ( $1 \mathrm{pH}$ unit relative to the cytosol) of mycobacterial phagosomes (Hackam et al., 1997), suggesting that the proton pump remains a direct albeit limited source of acid equivalents for mycobacterial phagosomes. This concept has been supported by studies

Table 1. Host cell markers on mycobacterial phagosomes. ${ }^{a}$

\begin{tabular}{|c|c|c|}
\hline & $\begin{array}{l}\text { Model } \\
\text { phagosomes }(\phi)^{\mathrm{b}}\end{array}$ & $\begin{array}{l}\text { Mycobacterial } \\
\text { phagosomes }^{\mathrm{C}}\end{array}$ \\
\hline $\mathrm{H}^{+}$-ATPase pump & + & $-^{d}$ \\
\hline $\mathrm{Na}^{+} / \mathrm{H}^{+}$exchanger & $+{ }^{e}$ & $N^{e}$ \\
\hline $\mathrm{Na}^{+} / \mathrm{K}^{+}$pumps & - & ND \\
\hline MHC class I & - & + \\
\hline MHC class II & - & + \\
\hline Cathepsin D & + & + \\
\hline Lamp-1 and -2 & + & $+/-^{f}$ \\
\hline TfR & $-{ }^{g}$ & $+^{g}$ \\
\hline Annexins & $+^{\mathrm{h}}$ & $+^{\mathrm{h}}$ \\
\hline Nramp1 & + & ND \\
\hline Rab5 & $++^{i}$ & $+^{i}$ \\
\hline Rab7 & + & $-j$ \\
\hline t-SNAREs ${ }^{k}$ & + & ND \\
\hline v-SNAREs ${ }^{k}$ & + & ND \\
\hline
\end{tabular}

a. Information based on: Clemens and Horwitz (1995); Clemens and Horwitz (1992); Clemens and Horwitz (1996); Desjardins et al. (1994); Desjardins et al. (1997); Hackam et al. (1996); Hackam et al. (1998); Strugill-Koszycki et al. (1996); Strugill-Koszycki et al. (1994); Via et al. (1997).

b. Model phagosomes $(\phi)$ : latex beads (1-20 h post phagocytosis). c. The current data on $M$. tuberculosis, $M$. bovis BCG and $M$. avium phagosomal compartments are in general agreement and are compiled here without making distinctions between different strains within the $M$. tuberculosis complex or between mycobacterial species that served as the source of specific information. This, however, does not preclude existence of major differences that remain to be delineated. d. $\Delta \mathrm{pH}$ of 1 unit in $M$. bovis BCG phagosomes relative to the cytosol can be abrogated by bafilomycin and conconamycin implicating traces of $\mathrm{H}^{+}$-ATPase activity in delivery of acid equivalents.

e. Detected by Western blots but exchange activity demonstrated only in reverse by functional assays. ND, not determined.

f. Lamp- 1 and 2 have been seen on mycobacterial phagosomes with variable intensity.

g. TfR persists on mycobacterial phagosomes while normally it recycles rapidly to the plasma membrane.

h. Annexins I-V on $\phi$ (annexin IV enriched on older $\phi)$. Annexins III, IV and V detected on M. tuberculosis H37Ra phagosomes in dendritic cells.

i. Rab5 accumulates on $M$. bovis BCG and is present on $\phi$.

j. Rab7 is not present on M. bovis BCG but succeeds Rab5 on $\phi$.

k. t-SNAREs, syntaxins 2, 3 and 4; v-SNAREs, synaptobrevins I and II. See text for cellubrevin. 
with permeabilized cells and acidification of $M$. bovis BCG phagosomes subsequent to the activation of vacuolar $\mathrm{H}^{+}$. ATPase by exogenously added cofactors (Hackam et al., 1998).

\section{Effects of cytokines and Nramp1 on phagosomal acidification}

The significance of the reduced acidification of $M$. tuberculosis phagosomes is multifaceted and may affect (i) killing/ survival of the microbe; (ii) vacuolar maturation processes, as the recruitment of at least some parts of membrane trafficking machinery may depend on vesicular $\mathrm{pH}$ (Aniento et al., 1996); and (iii) antigen processing and presentation. It appears that factors associated with host innate and adaptive immunity can modulate acidification of mycobacterial phagosomes. In cytokine-activated macrophages, e.g. murine bone marrow-derived macrophages or $\mathrm{J} 774$ cells, mycobacterial phagosomes acidify more readily upon treatment with interferon (IFN)- $\gamma$ and lipopolysaccharide (LPS) (Schaible et al., 1998; Via et al., 1998).

A role for the natural resistance-associated macrophage protein 1 (Nramp1; Bcg/lty/Lsh) has been demonstrated recently (Hackam et al., 1998) as directly or indirectly promoting $\mathrm{H}^{+}$-ATPase-dependent acidification of $M$. bovis BCG phagosomes in peritoneal macrophages obtained from $\mathrm{Bcg}^{\mathrm{r}}$ animals. In keeping with these observations, wildtype, functional Nramp1 is delivered to model phagosomes, whereas mutant forms are not detectable. Intriguingly, although Nramp1 mutations (in the absence of acquired immunity achieved by $T$ cell depletion) permit increased growth of $M$. bovis BCG in the lungs and spleens of the mutant $\mathrm{Bcg}{ }^{\mathrm{s}}$ mice relative to wild-type $\mathrm{Bcg}^{\mathrm{r}}$ animals (Medina and North, 1996a), Nrapm1-proficient $\left(\mathrm{Bcg}^{r}\right)$ animals appear unexpectedly more sensitive to infections with the virulent M. tuberculosis H37Rv (Medina and North, 1996b). Resolution of this paradox may provide insights into possible infection stage specific differences in intracellular environments experienced by the vaccine strain $M$. bovis BCG and virulent $M$. tuberculosis.

\section{Markers on mycobacterial phagosomes}

Mycobacterium tuberculosis and M. avium phagosome maturation is arrested at an early stage (Clemens and Horwitz, 1996; Strugill-Koszycki et al., 1996) characterized by the persistence of early endosomal markers and limited acquisition of late endosomal markers (Strugill-Koszycki et al., 1996). The majority of current insights into the makeup of mycobacterial phagosomes (Table 1) have originated from the laboratories of Drs Russell and Horwitz, who studied patterns of several endocytic markers on mycobacterial phagosomes. The M. tuberculosis phagosome lacks mannose 6-phosphate receptors, displays reduced clearance of MHC class I and II molecules, and stains for transferrin receptor (TfR) (Clemens and Horwitz, 1995). Both $M$. tuberculosis and $M$. avium phagosomes remain accessible to subsequently internalized transferrin (Clemens and Horwitz, 1996; Strugill-Koszycki et al., 1996) and to plasma membrane glycosphingolipids (Russell et al., 1996). These phagosomes appear to acquire limited amounts of major lysosome-associated membrane (glyco)proteins (Lamp-1 and Lamp-2) (Xu et al., 1994; Clemens and Horwitz, 1995) and cathepsin D (Clemens and Horwitz, 1995; Strugill-Koszycki et al., 1996). Increased Lamp-2 staining of $M$. bovis BCG phagosomes has been reported in resting $\mathrm{Bcg}^{r}$ macrophages relative to $\mathrm{Bcg}^{s}$ cells (Hackam et al., 1998).

M. tuberculosis phagosomes persistently stain for transferrin receptor at densities similar to those on the plasma membrane (Clemens, 1996), although recycling of transferrin and TfR represents an archetype of rapid removal of markers ( $t_{1 / 2}$ in minutes) from the endosome back to the plasma membrane. Intriguingly, the estimated $\mathrm{pH}$ of the $M$. avium phagosome stabilizes around 6.4, matching the average $\mathrm{pH}$ of the recently recognized perinuclear recycling endosomal compartment rich in TfR (Ullrich et al., 1996). Furthermore, studies by Russell and colleagues (Strugill-Koszycki et al., 1996) and Clemens and Horwitz (1996) indicate that the mycobacterial phagosome may have continual access to TfR located in the recycling endosome, whereas phagosomes in general (represented by IgG-coated beads) maintain only a transient access (for about $5 \mathrm{~min}$ ) to the same compartment. One perhaps oversimplified interpretation of such observations could be that the mycobacterial phagosome is arrested at a '5-min-old phagosome' stage locked in a state of continuous communication with the TfR recycling compartment.

According to some proposals (de Chastellier and Thilo, 1997), the delayed clearance of TfR and other plasma membrane markers may be linked to the tightly apposed membrane encasing the bacilli, resulting in round phagosomes with an apparent lack of tubular extensions that may be necessary for efficient recycling. However, the morphological features alone cannot explain the maturation arrest, as illustrated by experiments with mutant RhoD (a small GTPase that integrates endosomal membrane transport with the cytoskeleton) in which TfR recycling was not affected although endosomes lost their tubular processes and appeared spherical (Murphy et al., 1996). In addition, hydrophobic latex beads, which seem to assume similar geometry to mycobacterial phagosomes (de Chastellier and Thilo, 1997), show slow but complete maturation into the phagolysosome (Desjardins et al., 1994). Furthermore, passive surface properties of $M$. tuberculosis, $M$. bovis and $M$. avium may not be sufficient to define all intracellular events, as the fate of dead bacteria differs from that of live organisms. An alternative explanation for the presence of TfR on mycobacterial phagosomes has been offered, 
e.g. that these vesicles, because of their retention of the composition similar to the plasma membrane, continue to receive TfR (Hasan et al., 1997).

\section{Two-dimensional analyses of proteins on purified mycobacterial phagosomes}

Recently, new methodologies such as phagosome purification (Strugill-Koszycki et al., 1994; Hasan et al., 1997; Via et al., 1997) coupled with two-dimensional (2D) protein analysis have opened new avenues for investigations of mycobacterial phagosomes. The 2D patterns of stained or metabolically labelled polypeptides on $M$. avium and $M$. bovis BCG phagosomes have thus far indicated very few differences relative to IgG-coated bead phagosomes (Strugill-Koszycki et al., 1997) or endosomal organelles (Hasan et al., 1997). Nevertheless, the three polypeptides identified by Pieters and colleagues as specifically recruited to M. bovis BCG phagosomes (Hasan et al., 1997) may prove to be of significance. Nevertheless, the salient characteristics of the $M$. tuberculosis phagosome may depend on minor, regulatory components that cannot be readily visualized using conventional staining and detection techniques. Recently, 2D gel analyses (Deretic et al., 1997; Via et al., 1997) have been applied to study less abundant components on $M$. bovis BCG phagosomes, i.e. small GTP-binding proteins. These small GTPases from the Ras superfamily (Hall and Zerial, 1995), regulate critical cellular processes such as: (i) growth and differentiation, endoplasmic reticulum (ER) and Golgi transport, nuclear import/export (Ras, Sar and Ran subfamilies); (ii) co-ordination of membrane transport with cytokeletal functions, cell adhesion, motility and shape (Rho subfamily); and (iii) compartment-specific membrane and vesicular trafficking (ARF and Rab subfamilies).

Rab proteins are of direct relevance for phagosome interactions with the endosomal system that takes place during the maturation of these organelles into the phagolysosome (Desjardins et al., 1994). Because small GTPbinding proteins, unlike the large heterotrimeric $G$ proteins, can renature upon transfer to membrane after separation by SDS-PAGE, their composition and presence can be studied on purified phagosomal membrane by using highly sensitive methods using overlays with radiolabelled GTP and comparing autoradiograms with the existing $2 \mathrm{D}$ maps of Rab and Rho proteins (Huber et al., 1994). This has led to the identification of unique patterns of small GTPases on mycobacterial phagosomes (Via et al., 1997), including Rabs, that will be discussed in subsequent sections.

\section{Phagosomal maturation and membrane fusion machinery}

Phagosomes are dynamic structures (Desjardins et al.,
1994; 1997; Alvarez-Dominguez et al., 1996), which, upon formation, interact with various parts of the endosomal network exchanging patches of membrane and luminal contents (Desjardins et al., 1994), as summarized in the 'kiss and run' model (Desjardins, 1995). The sequential interaction of phagosomes with early and late endosomes culminates with phagosomal maturation into an organelle frequently referred to as the phagolysosome. All trafficking events involving vesicular transport of lipids and proteins between intracellular organelles in eukaryotic cells are highly controlled processes dependent on a multicomponent vesicle docking and fusion machinery. Typically, the fusion system is composed of: (i) ATPase NSF ( $\underline{N}$-ethylmaleimide-sensitive factor) and $\alpha$ SNAP (soluble NSF attachment protein), and (ii) integral membrane proteins $v$ - and t-SNAREs (vesicle and target membrane SNAP receptors), which serve as docking and fusion devices. NSF and SNAP are required for separation of $v$ - and $t$-SNAREs, which have a spontaneous tendency to form complexes. This segregation provides free energy liberated during the SNARE pairing and subsequent fusion steps (Weber et al., 1998). Both t-SNAREs (syntaxins 2, 3 and 4) and v-SNAREs (VAMP, synaptobrevins) have been detected on model phagosomes (Hackam et al., 1996; Desjardins et al., 1997). Recently, another v-SNARE, cellubrevin, has been detected on purified M. bovis BCG phagosomes (R. A. Fratti and V. Deretic, unpublished). Current studies are focusing on the status of $\mathrm{v}$ - and t-SNAREs on $M$. tuberculosis and $M$. bovis BCG phagosomes to determine whether changes in these molecules may help contribute to events underlying the mycobacterial phagosome maturation arrest.

\section{Rab proteins on mycobacterial phagosomes: accumulation of Rab5 and exclusion of Rab7}

When cognate SNAREs on two apposed membranes are paired, they form a structure termed SNAREpin (by analogy with viral hairpin fusion proteins) resulting in lipid bilayer fusion (Weber et al., 1998). However, membranes in eukaryotic cells do not fuse indiscriminately, and such processes are under close supervision by regulatory systems, including the small GTPase Rabs, which restrict uncontrolled intermixing of endovacuolar organelles and prevent their complete fusion or disintegration. The mechanism of how Rabs control membrane fusion is not completely clear. It is likely that these small G proteins, when in their active GTP-bound state, function at the docking stage, perhaps by displacing a negative regulator preventing v-SNARE-t-SNARE interactions, as recently documented in yeast (Lupashin and Waters, 1997). The major Rabs (Fig. 2) associated with various parts of the endosomal network are Rab4 (controlling fast recycling out of the early endosome); Rab5 (controlling endocytosis and homotypic fusions within the early endosome); Rab7 (localized

(C) 1999 Blackwell Science Ltd, Molecular Microbiology, 31, 1603-1609 


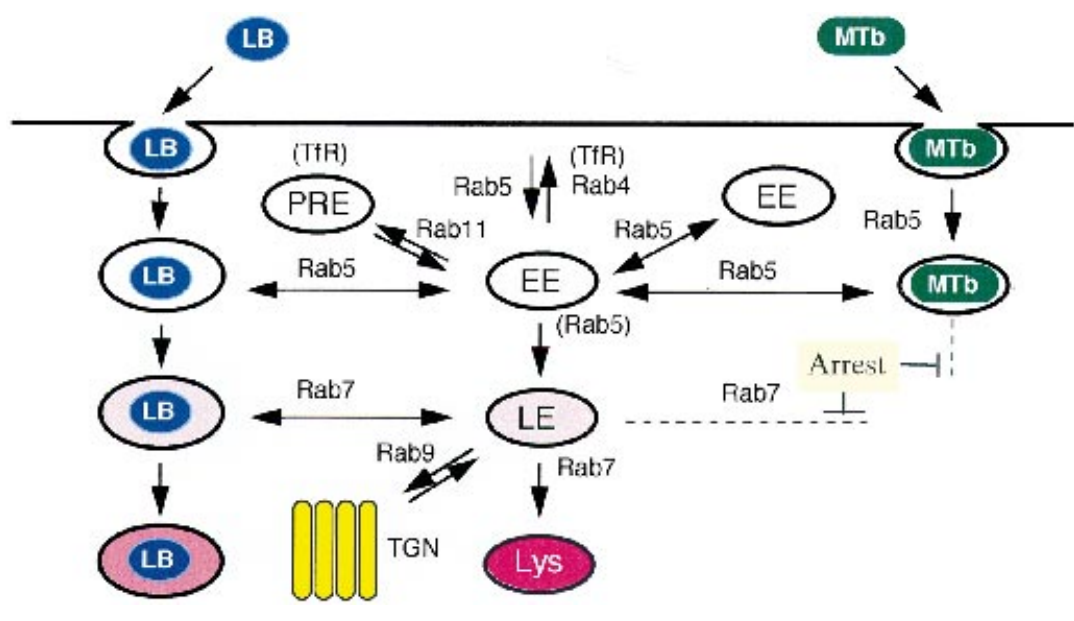

Fig. 2. Mycobacterium tuberculosis phagosome maturation arrest: accumulation of Rab5 and exclusion of Rab7. Rab proteins control membrane trafficking within endosomal compartments: Rab4, fast recycling branch of the sorting endosome (TfR, transferrin receptor); Rab5, endocytosis and homotypic fusions between early endosome (EE) (also newly formed phagosomes); (Rab5), recent studies (Jahraus et al., 1998) have shown that exogenously added Rab5 can stimulate fusion between newly formed phagosomes and late endocytic compartments in vitro; Rab7, late endosome (LE) (also mature phagosomes); Rab9, recycling from LE to trans Golgi network (TGN); Rab11, perinuclear recycling endosome (PRE). Mtb, $M$ tuberculosis; LB, latex beads. Green, M. tuberculosis. Different shades of red indicate increasing phagolysosomal characteristics. Dashed lines, block in M. tuberculosis phagosome maturation. to the late endosome); Rab9 (controlling endosome to TGN recycling); and Rab11 (localized in the pericentriolar/ paranuclear early endosomal recycling compartment).

Of particular importance for the M. tuberculosis phagosome are Rab5 and Rab7. Rab5 has been strongly implicated in the interactions between phagosomes and the endocytic pathway in macrophages (Desjardins et al., 1994; Desjardins, 1995; Alvarez-Dominguez et al., 1996; Via et al., 1997). Rab7 regulates late endosomal membrane trafficking (Press et al., 1998) and has been implicated in interactions between phagosomes and late endosomal compartments (Desjardins et al., 1994). We have recently defined the arrest of $M$. bovis BCG phagosome maturation as a block between stages controlled by Rab5 and Rab7 GTPases (Via et al., 1997) (Fig. 2). According to these findings, Rab5 accumulates on M. bovis BCG phagosomes, whereas Rab7 is not present on mycobacterial phagosomes. This is in contrast to model phagosomes with latex beads, which go through sequential acquisition of Rab5 and Rab7. Incidentally, expression in transfected cells of a dominant negative mutant, Rab7N125I (Press et al., 1998), which can no longer bind GTP, causes accumulation of late endosomal markers in early endosomes positive for TfR (mycobacterial phagosomes, which are blocked between the Rab5 and Rab7 stages, also accumulate TfR; see earlier sections). Moreover, Rab7N125I blocks transport to the late endosome and causes accumulation of cathepsin $\mathrm{D}$ (as a $46-48 \mathrm{kDa}$ intermediate form) in early endosomes (Press et al., 1998). Note that cathepsin D also accumulates in mycobacterial vacuoles as a $46-$ $48 \mathrm{kDa}$ processing intermediate (Strugill-Koszycki et al., 1996). Thus, the exclusion of Rab7 from M. bovis BCG phagosomes can explain at least in part the pattern of markers seen on mycobacterial phagosomal compartments (Table 1). The observed accumulation of Rab5 and exclusion of Rab7 define more precisely the check point that has been compromised in the maturation of mycobacterial phagosomes. These observations also provide potential molecular targets used by $M$. tuberculosis for interference with organelle biogenesis and membrane trafficking in the host.

\section{Rab effectors: a look ahead}

Factors interacting with Rab5 in mammalian cells have only recently begun to be identified and some of them have important functions such as Rab5 GTPase activating proteins (GAP, e.g. Tuberin), Rab5 guanine nucleotide exchange factors (GEF, e.g. Rabex-5) and Rab5 effectors (Rabaptin-5, Rabaptin-5 $\beta$ and the early endosomal autoantigen EEA1). These factors play important roles in cell physiology, as attested to by the fact that Tuberin is encoded by a tumour-suppressor gene, and that Rabaptin5 is essential for early endosomal function. EEA 1 is restricted in distribution to early endosomes where it is recruited by $\mathrm{PI}(3)$ kinase products and Rab5 and is believed to provide directionality to Rab5-dependent membrane trafficking (Simonsen et al., 1998). Rab5 is active in its GTP-bound form (Fig. 2), but only a small portion of Rab5 is bound to GTP at any given time. GTP hydrolysis downregulates Rab5 function and GTPase activity stimulated by GAP proteins diminishes Rab5 function in endocytosis (Xiao et al., 1997). Rabaptin-5 interacts with GTP-bound Rab5 and stabilizes it in its active conformation; it also provides a link between Rab5- and Rab4-dependent pathways as it has distinct domains for binding of both GTPases, possibly co-ordinating membrane flow in and out of early endosomes (Vitale et al., 1998). Interestingly, Rabaptin-5 is a substrate for caspase cleavage during apoptosis, thus linking programmed cell death with the attendant disintegration of the endocytic apparatus (Cosulich et al., 1997). As specific pathways of cell death including apoptosis have been implicated in the control of $M$. tuberculosis and M. bovis BCG in host macrophages (Molloy et al., 1994; 
Keane et al., 1997; Lammas et al., 1997), it is possible that changes in Rabaptin- 5 or other Rab-interacting molecules may affect mycobacterial phagosomes including a fusion with undesirable degradative compartments.

\section{Concluding remarks}

Ongoing investigations of the status of Rabs, SNAREs, and other components of the membrane fusion machinery in infected cells and on M. tuberculosis phagosomes, may help define the molecular mechanism used by the tubercle bacillus in constructing its intracellular niche to desired specifications. The current gap in our understanding of the fundamental cell biology phenomena downstream of the immune mechanisms deserves further attention, as cytokine-activated macrophages can overcome the maturation block and acidify the mycobacterial phagosome (Schaible et al., 1998; Via et al., 1998). The precise regulatory trafficking events involved in these phenomena remain to be determined. The hope is that such and related studies will delineate the molecular processes underlying mycobacterial phagosome maturation arrest and provide insights into how host defences (e.g. macrophage-activating cytokines and cytotoxic mechanisms) may affect and counteract such phenomena under conditions consistent with protective immune responses. Lastly, the intracellular compartments in which $M$. tuberculosis resides during the establishment of latency may have unique characteristics. Because latency is the predominant form of $M$. tuberculosis infections, a potential definition of intracellular environments in which the dormant bacilli persist may be necessary if we wish to understand how this microorganism manages to infect billions of people worldwide and intervene with its notorious success as a powerful human pathogen.

\section{Acknowledgements}

We thank D. Deretic for collaboration and discussions, and L. E. Via and E. Pagan-Ramos for scientific contributions and help with displays. This work was supported by National Institutes of Health grants Al35217 and Al42999.

\section{References}

Alvarez-Dominguez, C., Barbieri, A.M., Beron, W., Wandinger-Ness, A., and Stahl, P.D. (1996) Phagocytosed live Listeria monocytogenes influences Rab5-regulated in vitro phagosome-endosome fusion. J Biol Chem 271: 1383413843.

Aniento, F., Gu, F., Parton, R.G., and Gruenberg, J. (1996) An endosomal beta COP is involved in the $\mathrm{pH}$-dependent formation of transport vesicles destined for late endosomes. J Cell Biol 133: 29-41.

Armstrong, J.A., and Hart, P.D. (1971) Response of cultured macrophages to $M$. tuberculosis with observations of fusion of lysosomes with phagosomes. J Exp Med 134: 713-740. de Chastellier, C., and Thilo, L. (1997) Phagosome maturation and fusion with lysosomes in relation to surface property and size of the phagocytic particle. Eur J Cell Biol 74: 49-62.

Clemens, D.L. (1996) Characterization of the Mycobacterium tuberculosis phagosome. Trends Microbiol 4: 133-117.

Clemens, D.L., and Horwitz, M.A. (1992) Membrane sorting during phagocytosis: selective exclusion of major histocompatibility complex molecules but not complement receptor CR3 during conventional and coiling phagocytosis. J Exp Med 175: 1317-1326.

Clemens, D.L., and Horwitz, M.A. (1995) Characterization of the Mycobacterium tuberculosis phagosome and evidence that phagosomal maturation is inhibited. J Exp Med 181: 257-270.

Clemens, D.L., and Horwitz, M.A. (1996) The Mycobacterium tuberculosis phagosome interacts with early endosomes and is accessible to exogenously administered transferrin. $J$ Exp Med 184: 1349-1355.

Cosulich, S.C., Horiuchi, H., Zerial, M., Clarke, P.R., and Woodman, P.G. (1997) Cleavage of rabaptin-5 blocks endosome fusion during apoptosis. EMBO J 16: 6812-6191.

Crowle, A., Dahl, R., Ross, E., and May, M. (1991) Evidence that vesicles containing living virulent $M$. tuberculosis or $M$. avium in cultured human macrophages are not acidic. Infect Immun 59: 1823-1831.

De Camilli, P., Emr, S.D., McPherson, P.S., and Novick, P. (1996) Phosphoinositides as regulators of membrane traffic. Science 271: 1533-1539.

Deretic, V., Via, L.E., Fratti, R., and Deretic, D. (1997) Mycobacterial Phagosome Maturation, Rab Proteins, and Intracellular Trafficking. Electrophoresis 18: 2542-2547.

Desjardins, M. (1995) Biogenesis of phagolysosomes: the 'kiss and run' hypothesis. Trends Cell Biol 5: 183-186.

Desjardins, M., Huber, L.A., Parton, R.G.F., and Griffiths, G. (1994) Biogenesis of phagolysosomes proceeds through a sequential series of interactions with endocytic apparatus. J Cell Biol 124: 677-688.

Desjardins, M., Nzala, N.N., Corsini, R., and Rondeau, C. (1997) Maturation of phagosomes is accompanied by changes in their fusion properties and size-selective acquisition of solute materials from endosome. J Cell Sci 110: 2303-2314.

Ernst, J.D. (1998) Macrophage receptors for Mycobacterium tuberculosis. Infect Immun 66: 1277-1281.

Hackam, D.J., Rotstein, O.D., Bennett, M.K., Klip, A., Grinstein, S., and Manolson, M.F. (1996) Characterization and subcellular localization of target membrane soluble NSF attachment protein receptors (t-SNAREs) in macrophages. J Immunol 156: 4377-4383.

Hackam, D.J., Rotstein, O.D., Zhang, W.-J., Demaurex, N., Woodside, M., Tsai, O., and Grinstein, S. (1997) Regulation of phagosomal acidification. J Biol Chem 272: 2981029820.

Hackam, D.J., Rotstein, O.D., Zhang, W.-J., Gruenheid, S., Gros, P., and Grinstein, S. (1998) Host resistance to intracellular infection: mutation of natural resistance-associated macrophage protein 1 (Nramp1) impairs phagosomal acidification. J Exp Med 188: 351-364.

Hall, A., and Zerial, M. (1995) Overview of the Ras superfamily of small GTPases. In: Guidebook to the Small 
Gtpases. Zerial, M., and Huber, L.A. (ed.). Oxford: Oxford University Press, pp. 3-11.

Hasan, Z., Schlax, C., Kuhn, L., Lefkovits, I., Young, D., Thole, J., and Pieters, J. (1997) Isolation and characterization of the mycobacterial phagosome: segregation from the endosomal/lysosomal pathway. Mol Microbiol 24: 545553.

Huber, L.A., Ullrich, O., Takai, Y., Lutcke, A., Dupree, P., Olkkonen, V., et al. (1994) Mapping of Ras-related GTPbinding proteins by GTP overlay following two-dimensional gel electrophoresis. Proc Natl Acad Sci USA 91: 78747878.

Jahraus, A., Tjelle, T.E., Berg, T., Habermann, A., Storrie, B., Ulrich, O., and Griffiths, G. (1998) In vitro fusion of phagosomes with different endocytic organelles from J774 macrophages. J Biol Chem 46: 30379-30390.

Keane, J., Balcewicz-Sablinska, M.K., Remold, H.G., Chupp, G.L., Meek, B.B., Fenton, M.J., and Kornfeld, H. (1997) Infection by Mycobacterium tuberculosis promotes human alveolar macrophage apoptosis. Infect Immun 65: 298304.

Kusner, D.J., Hall, C.F., and Schlesinger, L.S. (1996) Activation of phospholipase $D$ is tightly coupled to the phagocytosis of Mycobacterium tuberculosis or opsonized zymosan by human macrophages. J Exp Med 184: 585-595.

Lammas, D.A., Stober, C., Harvey, C.J., Kendrick, N., Panchalingam, S., and Kumararatne, D.S. (1997) ATP-induced killing of mycobacteria by human macrophages is mediated by purinergic P2Z (P2X7) receptors. Immunity 7: 433-444.

Lupashin, V., and Waters, M.G. (1997) t-SNARE activation through transient interaction with a rab-like guanosine triphosphatase. Science 276: 1255-1258.

Medina, E., and North, R.A. (1996a) Mice that carry the resistance allele of the $B c g$ gene $\left(B c g^{r}\right)$ develop a superior capacity to stabilize bacille Calmette-Guerin (BCG) infections in their lungs and spleen over a protracted period in the absence of specific immunity. Clin Exp Immunol 104: 44-47.

Medina, E., and North, R.J. (1996b) Evidence incosistent with a role for the $\mathrm{Bcg}$ gene (Nramp1) in resistance of mice to infection with virulent Mycobacterium tuberculosis. $J$ Exp Med 183: 1045-1051.

Molloy, A., Laochumroonvorapong, P., and Kaplan, G. (1994) Apoptosis, but not necrosis, of infected monocytes is coupled with killing of intracellular bacillus CalmetteGuerin. J Exp Med 180: 1499-1509.

Murphy, C., Saffrich, R., Grummt, M., Gournier, H., Rybin, V., Rubino, M., et al. (1996) Endosome dynamics regulated by a rho protein. Nature 384: 427-432.

Oh, Y.-K., and Straubinger, R.M. (1996) Intracellular fate of Mycobacterium avium: use of dual-label spectrofluorometry to investigate the influence of bacterial viability and opsonization on phagosomal $\mathrm{pH}$ and phagosome-lysosome interaction. Infect Immun 64: 319-325.

Press, B., Feng, Y., Hoflack, B., and Wandinger-Ness, A. (1998) Mutant Rab7 causes the accumulation of cathepsin $\mathrm{D}$ and cation-independent mannose 6-phosphate receptor in an early endocytic compartment. J Cell Biol 140: 10751089.

Russell, D.G., Dang, J., and Strugill-Koszycki, S. (1996)
Mycobacterium avium- and Mycobacterium tuberculosiscontaining vacuoles are dynamic, fusion competent vesicles that are accessible to glycosphingolipids from the host cell plasmalemma. J Immunol 156: 4764-4773.

Schaible, U.E., Strugill-Koszycki, S., Schlesinger, P.H., and Russell, D.G. (1998) Cytokine activation leads to acidification and increases maturation of Mycobacterium aviumcontaining phagosomes in murine macrophages. $J$ Immunol 160: 1290-1296.

Schlesinger, L.S., Bellinger-Kawahara, C.G., Payne, N.R., and Horwitz, M.A. (1990) Phagocytosis of Mycobacterium tuberculosis is mediated by human monocyte complement receptors and complement component C3. J Immunol 144: 2771-2780.

Schorey, J.S., Carroll, M.C., and Brown, E.J. (1997) A macrophage invasion mechanism of pathogenic mycobacteria. Science 277: 1091-1093.

Simonsen, A., Lippe, R., Christoforidis, S., Gaullier, J.-M., Brech, A., Callaghan, J., et al. (1998) EEA1 links PI(3)K function to Rab5 regulation of endosome fusion. Nature 394: 494-498.

Strugill-Koszycki, S., Haddix, P.L., and Russell, D.G. (1997) The interaction between Mycobacterium and the macrophage analyzed by two-dimensional polyacrylamide gel electrophoresis. Electrophoresis 18: 2558-2565.

Strugill-Koszycki, S., Schaible, U.E., and Russell, D.G. (1996) Mycobacterium-containing phagosomes are accessible to early endosomes and reflect a transition state in normal phagosome biogenesis. EMBO J 15: 6960-6968.

Strugill-Koszycki, S., Schlesinger, P.H., Chakraborty, P., Haddix, P.L., Collins, H.L., Fok, A.K., et al. (1994) Lack of acidification in Mycobacterium phagosomes produced by exclusion of the vesicular proton-ATPase. Science 263: $678-681$.

Ullrich, O., Reinsch, S., Urbe, S., Zerial, M., and Parton, R.G. (1996) Rab11 regulates recycling through the pericentriolar recycling endosome. J Cell Biol 135: 913-924.

Via, L.E., Deretic, D., Ulmer, R.J., Hibler, N.S., Huber, L.A., and Deretic, V. (1997) Arrest of mycobacterial phagosome maturation is caused by a block in vesicle fusion between stages controlled by rab5 and rab7. J Biol Chem 272: 13326-13331.

Via, L.E., Fratti, R.A., McFalone, M., Pagan-Ramos, E., Deretic, D., and Deretic, V. (1998) Effects of cytokines on mycobacterial phagosome maturation. J Cell Sci 111: 897-905.

Vitale, G., Rybin, V., Savvas, C., Thornquist, P.-O., McCaffrey, M., Stenmark, H., and Zerial, M. (1998) Distinct Rab-binding domains mediate the interaction of Rabaptin-5 with GTPbound rab4 and rab5. EMBO J 17: 1941-1951.

Weber, T., Zemelman, B.V., McNew, J.A., Westermann, B., Gmachl, M., Parlati, F., et al. (1998) SNAREpins: minimal machinery for membrane fusion. Cell 92: 759-772.

Xiao, G.H., Shoarinejad, F., Jin, F., Golemis, E.A., and Yeung, R.S. (1997) The tuberous sclerosis 2 gene product, tuberin, functions as a Rab5 GTPase activating protein (GAP) in modulating endocytosis. J Biol Chem 272: 6097-6100.

Xu, S., Cooper, A., Sturgill-Koszycki, S., van Heyningen, T., Chaterjee, D., Orme, I., et al. (1994) Intracellular trafficking in Mycobacterium tuberculosis and Mycobacterium aviuminfected macrophages. J Immunol 153: 2568-2578. 